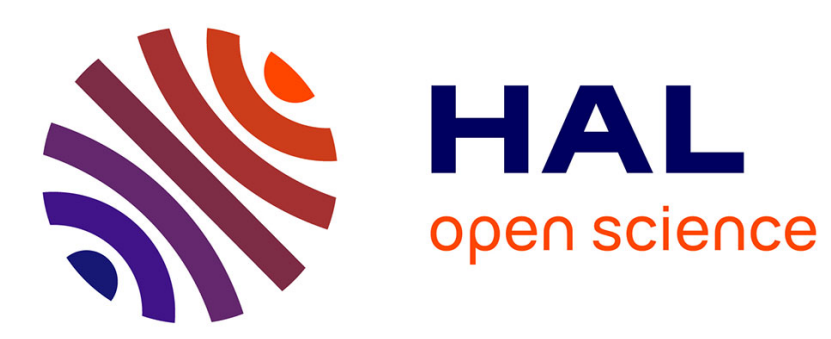

\title{
A statistical comparison between music and G-music
}

P. Vallet, Philippe Loubaton, X. Mestre

\section{To cite this version:}

P. Vallet, Philippe Loubaton, X. Mestre. A statistical comparison between music and G-music. ICASSP, Apr 2015, Brisbane, Australia. pp.A, 10.1109/ICASSP.2015.7178487 . hal-01618519

\section{HAL Id: hal-01618519 https://hal.science/hal-01618519}

Submitted on 18 Oct 2017

HAL is a multi-disciplinary open access archive for the deposit and dissemination of scientific research documents, whether they are published or not. The documents may come from teaching and research institutions in France or abroad, or from public or private research centers.
L'archive ouverte pluridisciplinaire HAL, est destinée au dépôt et à la diffusion de documents scientifiques de niveau recherche, publiés ou non, émanant des établissements d'enseignement et de recherche français ou étrangers, des laboratoires publics ou privés. 


\title{
A STATISTICAL COMPARISON BETWEEN MUSIC AND G-MUSIC
}

\author{
P. Vallet $^{1}$, P. Loubaton ${ }^{2}, X$. Mestre $^{3}$ \\ ${ }^{1}$ Laboratoire IMS (CNRS, Univ. Bordeaux, IPB), 351 Cours de la Libération, 33400 Talence, France \\ ${ }^{2}$ Laboratoire IGM (CNRS, Univ. Paris-Est/MLV), 5 Boulevard Descartes, 77454 Marne-la-Vallée, France \\ ${ }^{3}$ CTTC, Av. Carl Friedrich Gauss 08860 Castelldefels, Barcelona, Spain \\ pascal.vallet@ipb.fr, loubaton@univ-mlv.fr, xavier.mestre@cttc.cat
}

\begin{abstract}
This paper adresses the statistical performance of subspace DoA estimation using a sensor array, in the asymptotic regime where the number of samples and sensors both converge to infinity at the same rate. Improved subspace DoA estimators were derived (termed as G-MUSIC) in previous works, and were shown to be consistent and asymptotically Gaussian distributed in the case where the number of sources and their DoA remain fixed. In this case, which models widely spaced DoA scenarios, it is established that the traditional MUSIC method also provides consistent DoA estimates having the same asymptotic MSE as the G-MUSIC estimates. In the case of closely spaced DoA (i.e. with a spacing of the order of a beamwidth), it is shown that G-MUSIC is still able to consistently separate the sources, while it is no longer the case for MUSIC.
\end{abstract}

Index Terms - DoA estimation, MUSIC, consistency.

\section{INTRODUCTION}

The estimation of the Direction of Arrival (DoA) of plane waves impinging on an array of sensors is a fundamental problem in statistical signal processing and several methods have been developed since the past 40 years. In the context of $K$ narrow-band and far-field source signals received by an uniform linear array of $M>K$ sensors, the problem consists in estimating the DoA $\theta_{1}, \ldots, \theta_{K}$ from a set of $N$ (we assume $N \geq M$ for simplicity) observations $\mathbf{Y}_{N}=$ $\left[\mathbf{y}_{1}, \ldots, \mathbf{y}_{N}\right]$ modeled as

$$
\mathbf{Y}_{N}=\mathbf{A} \mathbf{S}_{N}+\mathbf{V}_{N}
$$

where

- $\mathbf{A}=\left[\mathbf{a}\left(\theta_{1}\right), \ldots, \mathbf{a}\left(\theta_{K}\right)\right]$ is the matrix of steering vectors, with

$$
\mathbf{a}(\theta)=\frac{1}{\sqrt{M}}\left[1, \ldots, \mathrm{e}^{\mathrm{i}(M-1) \theta}\right]^{T}
$$

- $\mathbf{S}_{N}$ contains the transmit source signals, considered as unknown deterministic ;

- $\mathbf{V}_{N}$ has i.i.d complex circular $\mathcal{N}\left(0, \sigma^{2}\right)$ entries, and represents a temporally and spatially white noise.

Among the most popular high resolution methods, the subspace algorithms, such as MUSIC [1], are widely used due to their reduced complexity since they involve a one-dimensional search over the set

This work has been partially supported by the French programs GDR ISIS/GRETSI "Jeunes Chercheurs" and Project ANR-12-MONU-OOO3 DIONISOS Project. of possible DoA, and are usually prefered over Maximum Likelihood which requires a multi-dimensional search. In particular, subspace methods are based in general on the observation that if the source signal matrix $\mathbf{S}_{N}$ is full rank $K$, the DoA $\theta_{1}, \ldots, \theta_{K}$ are the unique zeros of the pseudo-spectrum

$$
\eta(\theta)=\mathbf{a}(\theta)^{*} \boldsymbol{\Pi} \mathbf{a}(\theta),
$$

where $\boldsymbol{\Pi}_{N}$ is the orthogonal projection matrix onto the noise subspace, defined as the kernel of $N^{-1} \mathbf{A} \mathbf{S}_{N} \mathbf{S}_{N}^{*} \mathbf{A}^{*}$. In practice, $\boldsymbol{\Pi}_{N}$ is usually estimated from the so-called sample correlation matrix of the observations (SCM)

$$
\hat{\mathbf{R}}_{N}=\frac{\mathbf{Y}_{N} \mathbf{Y}_{N}^{*}}{N}=\frac{1}{N} \sum_{n=1}^{N} \mathbf{y}_{n} \mathbf{y}_{n}^{*},
$$

for which we will denote by $\hat{\lambda}_{1, N} \geq \ldots \geq \hat{\lambda}_{M, N}$ the eigenvalues and by $\hat{\mathbf{u}}_{1, N}, \ldots, \hat{\mathbf{u}}_{M, N}$ the eigenvectors. The MUSIC method consists in estimating $\theta_{1}, \ldots, \theta_{K}$ as the $K$ most significant minima of the estimated pseudo-spectrum ${ }^{1}$

$$
\hat{\eta}_{N}^{(t)}(\theta)=\mathbf{a}(\theta)^{*} \hat{\boldsymbol{\Pi}}_{N} \mathbf{a}(\theta),
$$

where $\hat{\boldsymbol{\Pi}}_{N}$ is the orthogonal projection matrix onto the eigenspace associated with the $M-K$ smallest eigenvalues of $\hat{\mathbf{R}}_{N}$.

It is also well-known that MUSIC methods (or subspace methods in general) suffer the so-called "threshold effect", which involves a severe degradation of performance when either the Signal to Noise Ratio (SNR) and/or the sample size $N$ are not large enough. In particular, this last situation may occur when the signal are short-time duration and/or stationary, and when the number of sensors $M$ is large. In that case, $M$ and $N$ may be of the same order of magnitude, and the usual statistical analysis of MUSIC, which assumed $N>>$ (see e.g. [2]), is irrelevant. One of the main reason is that MUSIC mainly relies on the SCM, which does not properly estimate the true covariance matrix $\mathbf{R}_{N}=\frac{1}{N} \mathbf{A} \mathbf{S}_{N} \mathbf{S}_{N}^{*} \mathbf{A}^{*}+\sigma^{2} \mathbf{I}$ in this context.

To model this more stringent scenario, [3] proposed to consider a new asymptotic regime in which both $M, N$ converges to infinity at the same rate, that is

$$
M, N \rightarrow \infty \text { such that } \frac{M}{N} \rightarrow c>0
$$

In particular, in this new asymptotic regime, the eigenvalues of $\hat{\mathbf{R}}_{N}$, instead of converging to the eigenvalues of $\mathbf{R}_{N}$, spread in several groups, involving a poor separation between the noise subspace and

\footnotetext{
${ }^{1}$ The superscript ${ }^{(t)}$ refers to "traditional estimate".
} 
its orthogonal complement the signal subspace. Based on results describing the behaviour of the eigenvalues and eigenvectors of large random matrices, an improved MUSIC DoA technique, termed as "G-MUSIC", was derived in [3] in the unconditional model case and later extended in [4] to the conditional model case.

In this paper, focused on the conditional case, we compare the statistical performance of MUSIC and G-MUSIC in two scenarios involving widely and closely spaced DoA. More precisely, we establish that

- when the number of sources $K$ and the corresponding DoA remain fixed as $M, N \rightarrow \infty$, while the "pseudo-spectrum" estimate of MUSIC is unconsistent, its minimization w.r.t. the DoA provides $N$-consistent ${ }^{2}$ estimates having the same asymptotic MSE as G-MUSIC.

- for two sources with an angular spacing of the order of a beamwidth, that is $\mathcal{O}\left(M^{-1}\right)$ as $M, N \rightarrow \infty$, G-MUSIC remains $N$-consistent while MUSIC is no more $N$-consistent, which means that MUSIC is no more able to asymptotically separate the DoA.

\section{THE G-MUSIC METHOD}

We consider for the remainder the doubly asymptotic regime where $M=M(N)$ is a function of $N$ such that the ratio $\frac{M}{N}$ converges to $c \in(0,1)$, and where $K$ is fixed and independent of $N$. In general, the DoA $\theta_{1, N}, \ldots, \theta_{K, N}$ may depend on $N$, and we also assume $\sup _{N}\left\|\mathbf{S}_{N}\right\|<\infty$, where $\|$.$\| stands for the spectral norm.$

The G-MUSIC method, described in [4], relies on the asymptotic theory of random matrices, from which we recall below some results. Let

$$
\hat{m}_{N}(z)=\frac{1}{M} \operatorname{tr}\left(\hat{\mathbf{R}}_{N}-z \mathbf{I}\right)^{-1}
$$

The function $z \mapsto \hat{m}_{N}(z)$ represents the Stieltjes transform of the empirical distribution $\hat{\mu}_{N}=\frac{1}{M} \sum_{k=1}^{M} \delta_{\hat{\lambda}_{k, N}}$ of the eigenvalues of the S.C.M. $\hat{\mathbf{R}}_{N}$, that is

$$
\hat{m}_{N}(z)=\int_{\mathbb{R}} \frac{\mathrm{d} \hat{\mu}_{N}(\lambda)}{\lambda-z} .
$$

From [5], for all $z \in \mathbb{C} \backslash \mathbb{R}^{+}, \hat{m}_{N}(z) \rightarrow m(z)$ a.s. as $N \rightarrow \infty$, where $m(z)=\int_{\mathbb{R}}(\lambda-z)^{-1} \mathrm{~d} \mu(\lambda)$ is the Stieltjes transform of the so-called Marcenko-Pastur distribution $\mu$, and which admits a density given by

$$
\frac{\mathrm{d} \mu(x)}{\mathrm{d} x}=\frac{\sqrt{\left(x-x^{-}\right)\left(x^{+}-x\right)}}{2 \sigma^{2} c \pi x} \mathbb{1}_{\left[x^{-}, x^{+}\right]}(x) .
$$

with $x^{-}=\sigma^{2}(1-\sqrt{c})^{2}$ and $x^{+}=\sigma^{2}(1+\sqrt{c})^{2}$. Moreover, $m(z)$ satisfies the following fundamental equation

$$
m(z)=\frac{1}{-z\left(1+\sigma^{2} c m(z)\right)+\sigma^{2}(1-c)} .
$$

Equivalently, with probability one, $\hat{\mu}_{N} \rightarrow \mu$ in distribution, that is, the empirical eigenvalue distribution of $\hat{\mathbf{R}}_{N}$ has the same asymptotic behaviour as the Marcenko-Pastur distribution.

\footnotetext{
${ }^{2} \mathrm{An}$ estimator $\hat{\theta}_{N}$ of a (possibly depending on $N$ ) DoA $\theta_{N}$ is defined as $N$-consistent if almost surely (a.s.), $N\left(\hat{\theta}_{N}-\theta\right) \rightarrow 0$, as $N \rightarrow \infty$.
}

Remark 1. The Marcenko-Pastur distribution was originally obtained as the limit distribution of the empirical eigenvalue distribution of the noise part $N^{-1} \mathbf{V}_{N} \mathbf{V}_{N}^{*}$. Nevertheless, since the rank $K$ of the deterministic perturbation $N^{-1} \mathbf{A} \mathbf{S}_{N} \mathbf{S}_{N}^{*} \mathbf{A}^{*}$ is independent of $N$ implies that the Marcenko-Pastur limit still holds for $\mathbf{R}_{N}$.

Under an additional assumption ensuring that the $K$ non-zero eigenvalues $\lambda_{k, 1} \geq \ldots \geq \lambda_{K, N}$ of $\frac{1}{N} \mathbf{A} \mathbf{S}_{N} \mathbf{S}_{N}^{*} \mathbf{A}^{*}$ are sufficiently separated from the $M-K$ zeros, we can describe the behaviour of the $\mathrm{K}$ largest eigenvalues of $\hat{\mathbf{R}}_{N}$.

Assumption 1. For $k=1, \ldots, K$, we have $\lambda_{k, N} \rightarrow \lambda_{k}$ as $N \rightarrow$ $\infty$, where $\lambda_{1}>\ldots>\lambda_{K}>\sigma^{2} \sqrt{c}$.

This condition, termed as subspace separation condition, also ensures that the range of $\frac{1}{N} \mathbf{A} \mathbf{S}_{N} \mathbf{S}_{N}^{*} \mathbf{A}^{*}$ is sufficiently separated from its kernel. Under the previous assumption, it is shown in [6] that for all $k=1, \ldots, K$,

$$
\hat{\lambda}_{k, N} \underset{N \rightarrow \infty}{\stackrel{p . s .}{\longrightarrow}} \phi\left(\lambda_{k}\right)=\frac{\left(\lambda_{k}+\sigma^{2} c\right)\left(\lambda_{k}+\sigma^{2}\right)}{\lambda_{k}},
$$

where $\phi\left(\lambda_{k}\right)>\sigma^{2}(1+\sqrt{c})^{2}$. Moreover, for all $\epsilon>0$,

$$
\hat{\lambda}_{K+1, N}, \ldots, \hat{\lambda}_{M, N} \in\left(\sigma^{2}(1-\sqrt{c})^{2}-\epsilon, \sigma^{2}(1+\sqrt{c})^{2} \epsilon\right),
$$

a.s. for $N$ large enough. Rephrased in another way, under the separation condition, the $K$ largest eigenvalues of $\boldsymbol{\Sigma}_{N} \boldsymbol{\Sigma}_{N}^{*}$ escape from the support of the Marcenko-Pastur distribution while the smallest $M-K$ eigenvalues are concentrated in a neighborhood of $\left[x^{-}, x^{+}\right]$.

Under the previous assumption, we also have results on the behaviour of the eigenvectors associated with $\hat{\lambda}_{1, N}, \ldots, \hat{\lambda}_{K, N}$; for all deterministic sequence $\left(\mathbf{d}_{N}\right)$ of unitary vectors,

$$
\left|\mathbf{d}_{N}^{*} \hat{\mathbf{u}}_{k, N}\right|^{2}=h\left(\phi\left(\lambda_{k}\right)\right)\left|\mathbf{d}_{N}^{*} \mathbf{u}_{k, N}\right|^{2}+o(1) \quad \text { a.s. }
$$

where

$$
h(z)=\frac{m(z)^{2}(c z m(z)-(1-c))}{c m(z)^{2}+2 c z m^{\prime}(z) m(z)-(1-c) m^{\prime}(z)} .
$$

Using algebric relations between $m\left(\phi\left(\lambda_{k}\right)\right)$ and $\lambda_{k}$, one can obtain the explicit formula

$$
h\left(\phi\left(\lambda_{k}\right)\right)=\frac{\lambda_{k}^{2}-\sigma^{4} c}{\lambda_{k}\left(\lambda_{k}+\sigma^{2} c\right)} .
$$

The convergence (3) is the keystone of the G-MUSIC method. Indeed, by taking $\mathbf{d}_{N}=\mathbf{a}(\theta)$, and using the fact that $\phi\left(\lambda_{k}\right)=\hat{\lambda}_{k, N}+$ $o(1)$ a.s., we obtain

$$
\eta_{N}(\theta)=1-\sum_{k=1}^{K}\left|\mathbf{a}(\theta)^{*} \mathbf{u}_{k, N}\right|^{2}=\hat{\eta}_{N}(\theta)+o(1),
$$

where

$$
\hat{\eta}_{N}(\theta)=1-\sum_{k=1}^{K} \frac{1}{h\left(\hat{\lambda}_{k, N}\right)}\left|\mathbf{a}(\theta)^{*} \hat{\mathbf{u}}_{k, N}\right|^{2} .
$$

Therefore, $\hat{\eta}_{N}(\theta)$ is a consistent estimator of the pseudo-spectrum $\eta_{N}(\theta)$, for all fixed $\theta$, in the doubly asymptotic regime considered above. The G-MUSIC method then consists in estimating the DoA by considering the $\mathrm{K}$ deepest local minima of $\theta \mapsto \hat{\eta}_{N}(\theta)$.

The consistency of these DoA estimates, which is the object of the next section, requires a stronger result on the following uniform convergence of the pseudo-spectrum, that is proved in [7].

$$
\left.\sup _{\theta \in[-\pi, \pi]}|| \mathbf{a}(\theta)^{*} \hat{\mathbf{u}}_{k, N}\right|^{2}-h\left(\phi\left(\lambda_{k}\right)\right)\left|\mathbf{a}(\theta)^{*} \mathbf{u}_{k, N}\right|^{2} \mid \underset{N \rightarrow \infty}{\stackrel{a . s .}{\longrightarrow}} 0 .
$$




\section{MUSIC AND G-MUSIC FOR WIDELY SPACED DOA}

In this section, we consider a widely spaced DoA situation, which occurs in practice when the DoA have an angular separation much larger than a beamwidth $\frac{2 \pi}{M}$. Mathematically speaking, we consider that the DoA $\theta_{1}, \ldots, \theta_{K}$ are fixed with respect to $N$. In that case, $\mathbf{A}^{*} \mathbf{A} \rightarrow \mathbf{I}$ and the separation condition holds if and only if the eigenvalues of $\frac{\mathbf{S}_{N} \mathbf{S}_{N}^{*}}{N}$ converge to $\lambda_{1}>\ldots>\lambda_{K}>\sigma^{2} \sqrt{c}$, which we will assume for the remainder of this section.

Let $\mathcal{I}_{1}, \ldots, \mathcal{I}_{K} \subset[-\pi, \pi]$ be $K$ compact disjoint intervals such that $\theta_{k} \in \operatorname{Int}\left(\mathcal{I}_{k}\right)$, where $\operatorname{Int}\left(\mathcal{I}_{k}\right)$ is the interior of $\mathcal{I}_{k}$. We define formally the G-MUSIC and MUSIC DoA estimators as

$$
\hat{\theta}_{k, N}=\underset{\theta \in \mathcal{I}_{k}}{\operatorname{argmin}} \hat{\eta}_{N}(\theta) \quad \text { and } \quad \hat{\theta}_{k, N}^{(t)}=\underset{\theta \in \mathcal{I}_{k}}{\operatorname{argmin}} \hat{\eta}_{N}^{(t)}(\theta) .
$$

Theorem 1. Under the separation condition, for $k=1, \ldots, K$,

$$
N\left(\hat{\theta}_{k, N}-\theta_{k}\right) \underset{N \rightarrow \infty}{\stackrel{a . s .}{\longrightarrow}} 0 \text { and } N\left(\hat{\theta}_{k, N}^{(t)}-\theta_{k}\right) \underset{N \rightarrow \infty}{\stackrel{a . s .}{\longrightarrow}} 0 .
$$

Proof. The $N$-consistency of G-MUSIC is already established in [7], and we prove hereafter the $N$-consistency of MUSIC.

From (6), we easily obtain that

$$
\sup _{\theta \in[-\pi, \pi]}\left|\hat{\eta}_{N}^{(t)}(\theta)-\eta_{N}^{(t)}(\theta)\right| \underset{N \rightarrow \infty}{\stackrel{a . s .}{\longrightarrow}} 0 .
$$

where

$$
\eta_{N}^{(t)}(\theta)=1-\mathbf{a}(\theta)^{*} \mathbf{U}_{N} \mathbf{D} \mathbf{U}_{N}^{*} \mathbf{a}(\theta),
$$

with $\mathbf{U}_{N}=\left[\mathbf{u}_{1, N}, \ldots, \mathbf{u}_{K, N}\right], \mathbf{D}=\operatorname{diag}\left(d_{1}, \ldots, d_{K}\right)$ and

$$
d_{k}=\frac{\lambda_{k}^{2}-\sigma^{4} c}{\lambda_{k}\left(\lambda_{k}+\sigma^{2} c\right)}
$$

Using the fact that $\mathbf{U}_{N}$ and $\mathbf{A}$ share the same image, we have

$$
\mathbf{U}_{N}=\mathbf{A}\left(\mathbf{A}^{*} \mathbf{A}\right)^{-1 / 2} \mathbf{V}_{N},
$$

where $\mathbf{V}_{N}$ is a $K \times K$ unitary matrix. Since $\mathbf{A}^{*} \mathbf{A} \rightarrow \mathbf{I}_{K}$ and

$$
\sup _{\theta \in \mathcal{I}_{k}}\left|\mathbf{a}(\theta)^{*} \mathbf{a}\left(\theta_{\ell}\right)\right| \rightarrow 0
$$

for all $\ell \neq k$, as $N \rightarrow \infty$, we obtain for all $k=1, \ldots, K$,

$$
\sup _{\theta \in \mathcal{I}_{k}}\left|\eta_{N}^{(t)}(\theta)-\left(1-\left|\mathbf{a}(\theta)^{*} \mathbf{a}\left(\theta_{k}\right)\right|^{2}\left\|\mathbf{D}^{1 / 2} \mathbf{V}_{N}^{*} \mathbf{e}_{k}\right\|^{2}\right)\right| \underset{N \rightarrow \infty}{\longrightarrow} 0
$$

Moreover, it holds that $\sup _{\theta \notin \cup_{k} \mathcal{I}_{k}} \eta_{N}^{(t)}(\theta) \underset{N \rightarrow \infty}{\longrightarrow} 1$. As the function $\theta \mapsto\left|\mathbf{a}(\theta)^{*} \mathbf{a}\left(\theta_{k}\right)\right|^{2}$ has a unique global maximum at $\theta_{k}$, we deduce that

$$
\hat{\theta}_{k, N}^{(t)} \underset{N \rightarrow \infty}{\stackrel{a . s .}{\longrightarrow}} \theta_{k} .
$$

We now improve (10) by showing the $N$-consistency, and for that purpose we follow the approach of [8] (also used in [7, Sec. 4]).

By definition, we have

$$
\begin{aligned}
\eta_{N}^{(t)}\left(\hat{\theta}_{k, N}^{(t)}\right) & \leq\left|\eta_{N}^{(t)}\left(\hat{\theta}_{k, N}^{(t)}\right)-\hat{\eta}_{N}^{(t)}\left(\hat{\theta}_{k, N}^{(t)}\right)\right|+\hat{\eta}_{N}^{(t)}\left(\hat{\theta}_{k, N}^{(t)}\right) \\
& \leq \sup _{\theta \in[-\pi, \pi]}\left|\eta_{N}^{(t)}(\theta)-\hat{\eta}_{N}^{(t)}(\theta)\right|+\hat{\eta}_{N}^{(t)}\left(\theta_{k}\right),
\end{aligned}
$$

and from (8), we obtain

$$
\begin{aligned}
\limsup _{N \rightarrow \infty} \eta_{N}^{(t)}\left(\hat{\theta}_{k, N}^{(t)}\right) & \leq \limsup _{N \rightarrow \infty} \hat{\eta}_{N}^{(t)}\left(\theta_{k}\right) \\
& =1-\liminf _{N \rightarrow \infty}\left\|\mathbf{D}^{1 / 2} \mathbf{V}_{N}^{*} \mathbf{e}_{k}\right\|^{2} \\
& <1
\end{aligned}
$$

since $\left\|\mathbf{D}^{1 / 2} \mathbf{V}_{N}^{*} \mathbf{e}_{k}\right\|^{2} \geq d_{K}>0$. Assume that the sequence $N\left(\hat{\theta}_{k, N}^{(t)}-\theta_{k}\right)$ is not bounded. Then we can extract a subsequence $\varphi(N)\left(\hat{\theta}_{k, \varphi(N)}^{(t)}-\theta_{k}\right)$ such that $\varphi(N)\left|\hat{\theta}_{k, \varphi(N)}^{(t)}-\theta_{k}\right| \underset{N \rightarrow \infty}{\longrightarrow} \infty$. This implies that $\eta_{\varphi(N)}^{(t)}\left(\hat{\theta}_{k, \varphi(N)}^{(t)}\right) \rightarrow 1$, a contradiction with (11). Since $N\left(\hat{\theta}_{k, N}^{(t)}-\theta_{k}\right)$ is bounded, we can extract a subsequence such that $\varphi(N)\left|\hat{\theta}_{k, \varphi(N)}^{(t)}-\theta_{k}\right| \underset{N \rightarrow \infty}{\longrightarrow} \beta$. If $\beta \neq 0$, then (9) gives

$$
\eta_{\varphi(N)}^{(t)}\left(\hat{\theta}_{k, \varphi(N)}^{(t)}\right)=1-\mathbf{e}_{k}^{*} \mathbf{V}_{N} \mathbf{D V}_{N}^{*} \mathbf{e}_{k} \operatorname{sinc}(\beta c / 2)^{2}+o(1)
$$

with probability one, where $\operatorname{sinc}(x)=\sin (x) / x$ if $x \neq 0$ and $\operatorname{sinc}(0)=1$. Since in that case,

$$
\limsup _{N \rightarrow \infty} \eta_{N}^{(t)}\left(\hat{\theta}_{k, N}^{(t)}\right)>1-\liminf _{N \rightarrow \infty} \mathbf{e}_{k}^{*} \mathbf{V}_{N} \mathbf{D V}_{N}^{*} \mathbf{e}_{k},
$$

this contradicts (11) again. Therefore all converging subsequences of the bounded sequence $N\left(\hat{\theta}_{k, N}^{(t)}-\theta_{k}\right)$ have the same limit, which is 0 , and thus the whole sequence converges itself to 0 , which concludes the proof.

By Theorem 1, MUSIC and G-MUSIC have the same first order behaviour, for widely spaced source DoA. In fact, we can also prove that this similarity holds at the 2 nd order, for asymptotically uncorrelated sources, thanks to the following result whose proof is omitted due to space constraints.

Theorem 2. Under the separation condition, and if $N^{-1} \mathbf{S}_{N} \mathbf{S}_{N}^{*} \rightarrow$ $\operatorname{diag}\left(\lambda_{1}, \ldots, \lambda_{K}\right)$,

$$
N^{3 / 2}\left(\hat{\theta}_{k, N}-\theta_{k}\right) \underset{N \rightarrow \infty}{\stackrel{\mathcal{D}}{\longrightarrow}} \mathcal{N}\left(0, \frac{6}{c^{2}} \frac{\sigma^{2}\left(\lambda_{k}+\sigma^{2}\right)}{\lambda_{k}^{2}-\sigma^{4} c}\right),
$$

and

$$
N^{3 / 2}\left(\hat{\theta}_{k, N}^{(t)}-\theta_{k}\right) \underset{N \rightarrow \infty}{\stackrel{\mathcal{D}}{\longrightarrow}} \mathcal{N}\left(0, \frac{6}{c^{2}} \frac{\sigma^{2}\left(\lambda_{k}+\sigma^{2}\right)}{\lambda_{k}^{2}-\sigma^{4} c}\right) .
$$

The results of Theorem 1 and 2 are illustrated in Figure 1, where we compared the empirical MSE of $\hat{\theta}_{1, N}$ together with its theoretical MSE given in Theorem 2 and the empirical MSE of $\hat{\theta}_{1, N}^{(t)}$. The parameters are $M=40, N=80$, and $\theta_{1}=0, \theta_{2}=5 \times \frac{2 \pi}{M}$. The signal matrix $\mathbf{S}_{N}$ has standard i.i.d $\mathcal{N}_{\mathbb{C}}(0,1)$ entries, and the separation condition occurs around $\mathrm{SNR}=0 \mathrm{~dB}$.

\section{MUSIC AND G-MUSIC FOR CLOSELY SPACED DOA}

In this section, we consider a closely spaced DoA scenario, where we let the DoA $\theta_{1, N}, \ldots, \theta_{K, N}$ depends on $N$ and converge to the same value at rate $\mathcal{O}\left(\frac{1}{M}\right)$. To simplify the presentation, we only consider $K=2$ sources with DoA $\theta_{1, N}$ and $\theta_{2, N}=\theta_{1, N}+\frac{\alpha}{N}$, where $\alpha>0$, and assume asymptotic uncorrelated sources with equal powers, that 


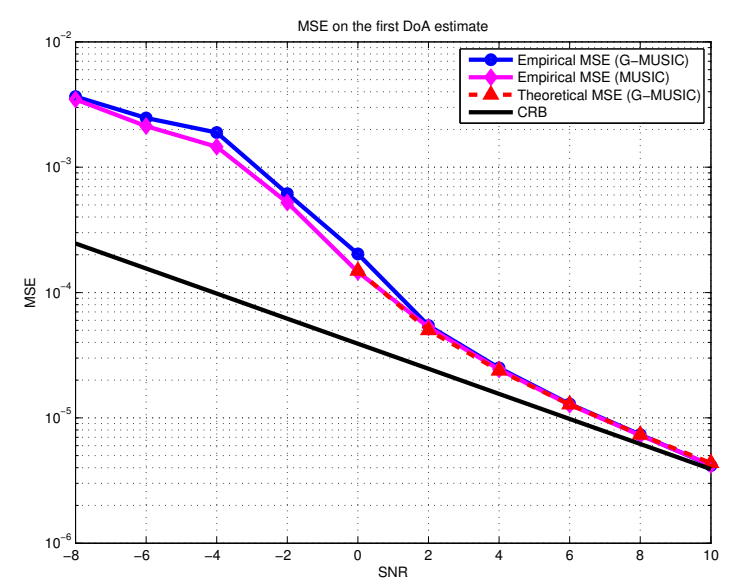

Fig. 1. Empirical MSE of $\hat{\theta}_{1, N}$ and $\hat{\theta}_{1, N}^{(t)}$ for widely spaced DoA

is $N^{-1} \mathbf{S}_{N} \mathbf{S}_{N}^{*} \rightarrow \mathbf{I}$. In this case, it is easily seen that the two non zero eigenvalues of $\frac{\mathbf{A S}_{N} \mathbf{S}_{N}^{*} \mathbf{A}^{*}}{N}$ converge to

$$
\lambda_{1}=1+|\operatorname{sinc}(\alpha c / 2)| \quad \text { and } \quad \lambda_{2}=1-|\operatorname{sinc}(\alpha c / 2)| .
$$

and the subspace separation condition holds iff $\lambda_{2}>\sigma^{2} \sqrt{c}$.

Since the DoA are not fixed with respect to $N$, we define, in the same way as (7), the G-MUSIC and MUSIC DoA estimates as

$$
\hat{\theta}_{k, N}=\underset{\theta \in \mathcal{I}_{k, N}}{\operatorname{argmin}} \hat{\eta}_{N}(\theta) \quad \text { and } \quad \hat{\theta}_{k, N}^{(t)}=\underset{\theta \in \mathcal{I}_{k, N}}{\operatorname{argmin}} \hat{\eta}_{N}^{(t)}(\theta)
$$

where $\mathcal{I}_{k, N}$ is defined, for $\epsilon \in(0, \alpha)$, as the compact interval

$$
\mathcal{I}_{k, N}=\left[\theta_{k, N}-\frac{\alpha-\epsilon}{2 N}, \theta_{k, N}+\frac{\alpha-\epsilon}{2 N}\right],
$$

Theorem 3. Under the separation condition and the close DoA scenario, for $k=1,2$,

$$
N\left(\hat{\theta}_{k, N}-\theta_{k, N}\right) \underset{N \rightarrow \infty}{\stackrel{a . s .}{\longrightarrow}} 0 .
$$

Moreover, there exists values of $\alpha$ such that $N\left(\hat{\theta}_{k, N}^{(t)}-\theta_{k, N}\right)$ does not converge to 0 .

Proof. Recall from (6) that we have $\sup _{\theta}\left|\hat{\eta}_{N}(\theta)-\eta_{N}(\theta)\right| \rightarrow_{N} 0$ with probability one, with

$$
\eta_{N}(\theta)=\mathbf{a}(\theta)^{*} \boldsymbol{\Pi}_{N} \mathbf{a}(\theta)=1-\mathbf{a}(\theta)^{*} \mathbf{A}\left(\mathbf{A}^{*} \mathbf{A}\right)^{-1} \mathbf{A}^{*} \mathbf{a}(\theta) .
$$

Like for (11), we have

$$
\limsup _{N \rightarrow \infty}\left|\hat{\eta}_{N}\left(\hat{\theta}_{k, N}\right)\right| \leq \limsup _{N \rightarrow \infty}\left|\hat{\eta}_{N}\left(\theta_{k, N}\right)\right|=0 .
$$

The key of the proof relies on the property that if $\left(\psi_{N}\right)$ is a sequence of angles,

$$
\eta_{N}\left(\psi_{N}\right) \rightarrow\left\{\begin{array}{ll}
1 & \text { if } N\left|\psi_{N}-\theta_{k, N}\right| \rightarrow \infty \\
1-\kappa(\beta) & \text { if } N\left(\psi_{N}-\theta_{k, N}\right) \rightarrow \beta
\end{array},\right.
$$

where

$$
\begin{aligned}
\kappa(\beta)= & \frac{\operatorname{sinc}(\beta c / 2)^{2}+\operatorname{sinc}((\beta-\alpha) c / 2)^{2}}{1-\operatorname{sinc}(\alpha c / 2)^{2}} \\
& -\frac{2 \operatorname{sinc}(\alpha c / 2) \operatorname{sinc}(\beta c / 2) \operatorname{sinc}((\beta-\alpha) c / 2)}{1-\operatorname{sinc}(\alpha c / 2)^{2}} .
\end{aligned}
$$

Morever, $\kappa(\beta) \leq 1$ with equality iff $\beta=0$ or $\beta=\alpha$. Therefore, if a subsequence of $N\left(\hat{\theta}_{k, N}-\theta_{k, N}\right)$ converges to $\beta$, we get thanks to (13) and (14), that $\beta=0$ necessarily, which proves the $N$-consistency of G-MUSIC. By doing the same way for MUSIC, we obtain for a sequence of angles $\left(\psi_{N}\right)$,

$$
\eta_{N}^{(t)}\left(\psi_{N}\right) \rightarrow \begin{cases}1 & \text { if } N\left|\psi_{N}-\theta_{k, N}\right| \rightarrow \infty \\ 1-\kappa^{(t)}(\beta) & \text { if } N\left(\psi_{N}-\theta_{k, N}\right) \rightarrow \beta\end{cases}
$$

where

$$
\begin{aligned}
\kappa^{(t)}(\beta)=( & \operatorname{sinc}(\beta c / 2)-\operatorname{sinc}((\beta-\alpha) c / 2))^{2} \frac{d_{1}(\alpha)}{2(1-\operatorname{sinc}(\alpha c / 2))} \\
& +(\operatorname{sinc}(\beta c / 2)+\operatorname{sinc}((\beta-\alpha) c / 2))^{2} \frac{d_{2}(\alpha)}{2(1+\operatorname{sinc}(\alpha c / 2))},
\end{aligned}
$$

with

$$
\begin{aligned}
d_{1}(\alpha) & =\frac{(1-\operatorname{sinc}(\alpha c / 2))^{2}-\sigma^{4} c}{(1-\operatorname{sinc}(\alpha c / 2))\left(1-\operatorname{sinc}(\alpha c / 2)+\sigma^{2} c\right)} \\
d_{2}(\alpha) & =\frac{(1+\operatorname{sinc}(\alpha c / 2))^{2}-\sigma^{4} c}{(1+\operatorname{sinc}(\alpha c / 2))\left(1+\operatorname{sinc}(\alpha c / 2)+\sigma^{2} c\right)} .
\end{aligned}
$$

Function $\kappa^{(t)}(\beta)$ does not admit in general a maximum at $\beta=0, \alpha$. As for (13), we can show that for all $|\beta|<\alpha / 2$, and all sequence $\left(\psi_{N}\right)$ such that $N\left(\psi_{N}-\theta_{k, N}\right) \rightarrow \beta$,

$$
\limsup _{N \rightarrow \infty}\left|\eta_{N}^{(t)}\left(\hat{\theta}_{k, N}^{(t)}\right)\right| \leq \limsup _{N \rightarrow \infty}\left|\hat{\eta}_{N}^{(t)}\left(\psi_{N}\right)\right| \leq 1-\kappa^{(t)}(\beta) .
$$

If $N\left(\hat{\theta}_{k, N}^{(t)}-\theta_{k, N}\right) \rightarrow 0$, then $\eta_{N}^{(t)}\left(\hat{\theta}_{k, N}^{(t)}\right) \rightarrow 1-\kappa^{(t)}(0)$, which contradicts (15), since in general, it may exist some values of $\alpha$ for which 0 is not maximum of $\kappa^{(t)}(\beta)$ on the interval $\left[-\frac{\alpha}{2}, \frac{\alpha}{2}\right]$.

The result of Theorem 3 is illustrated in Figure 2 where we use the same parameters as in Figure 1, except for the DoA set to $\theta_{1}=$ $0, \theta_{2}=\frac{\pi}{2 M}$. We can observe a difference of $4 \mathrm{~dB}$ between the threshold points of G-MUSIC and MUSIC.

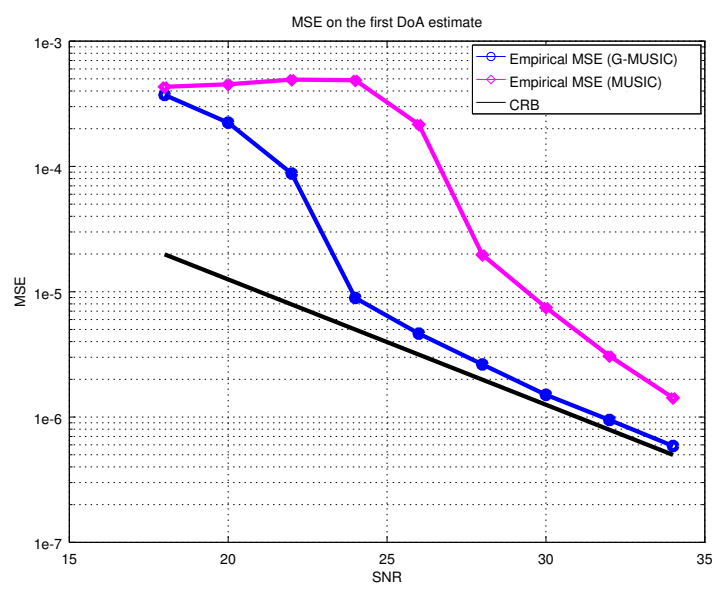

Fig. 2. Empirical MSE of $\hat{\theta}_{1, N}$ and $\hat{\theta}_{1, N}^{(t)}$ for closely spaced DoA 


\section{REFERENCES}

[1] R. Schmidt, "Multiple emitter location and signal parameter estimation," IEEE Transactions on Antennas and Propagation, vol. 34, no. 3, pp. 276-280, 1986.

[2] P. Stoica and A. Nehorai, "Music, maximum likelihood, and cramer-rao bound," Acoustics, Speech and Signal Processing, IEEE Transactions on, vol. 37, no. 5, pp. 720-741, 1989.

[3] X. Mestre and M.Á. Lagunas, "Modified subspace algorithms for doa estimation with large arrays," IEEE Transactions on Signal Processing, vol. 56, no. 2, pp. 598-614, 2008.

[4] P. Vallet, P. Loubaton, and X. Mestre, "Improved Subspace Estimation for Multivariate Observations of High Dimension: The Deterministic Signal Case," IEEE Transactions on Information Theory, vol. 58, no. 2, Feb. 2012, arXiv: 1002.3234.

[5] V.A. Marcenko and L.A. Pastur, "Distribution of eigenvalues for some sets of random matrices," Mathematics of the USSRSbornik, vol. 1, pp. 457, 1967.

[6] F. Benaych-Georges and R.R. Nadakuditi, "The singular values and vectors of low rank perturbations of large rectangular random matrices," To appear in Journal of Multivariate Analysis, 2011.

[7] W. Hachem, P. Loubaton, X. Mestre, J. Najim, and P. Vallet, "Large information plus noise random matrix models and consistent subspace estimation in large sensor networks," Random Matrices: Theory and Applications, vol. 1, no. 2, 2012.

[8] E.J. Hannan, “The estimation of frequency," Journal of Applied probability, vol. 10, no. 3, pp. 510-519, 1973. 